\title{
L'émergence du standard minimum de traitement des étrangers en droit international
}

Retour sur une question coloniale oubliée

The emergence of the minimum standard for the treatment of foreigners in

international law

Milcar Jeff Dorce

\section{OpenEdition}

Journals

Édition électronique

URL : https://journals.openedition.org/add/1747

DOI : $10.4000 /$ add. 1747

ISSN : 2606-1988

\section{Éditeur}

Presses universitaires de Rouen et du Havre

Édition imprimée

Date de publication : 1 juin 2020

Pagination : 9-36

ISBN : 979-10-240-1442-5

ISSN : 1955-0855

Référence électronique

Milcar Jeff Dorce, «L'émergence du standard minimum de traitement des étrangers en droit international », Les Annales de droit [En ligne], 14 | 2020, mis en ligne le 01 juin 2021, consulté le 12 janvier 2023. URL : http://journals.openedition.org/add/1747 ; DOl : https://doi.org/10.4000/add.1747

Ce document a été généré automatiquement le 12 janvier 2023.

Tous droits réservés 


\section{L'émergence du standard minimum de traitement des étrangers en droit international}

Retour sur une question coloniale oubliée

The emergence of the minimum standard for the treatment of foreigners in international law

Milcar Jeff Dorce

1 L'élaboration du standard minimum de traitement des étrangers par les juristes positivistes remonte au moment de l'universalisation du droit international - entre la fin $d u x x^{e}$ et le début $d u x^{e}$ siècle -, c'est-à-dire en plein contexte d'expansion impériale ${ }^{1}$. Du point de vue de l'histoire critique du droit international, cela revêt une double implication: d'une part, l'idée d'un droit international universel traduit la conquête des peuples non européens, ce qui revient pour les peuples européens à en tirer des avantages économiques et politiques² ; d'autre part, l'« âge de l'empire» européen, selon l'expression de Eric Hobsbawm ${ }^{3}$, a abouti à l'assimilation des cultures non européennes dans un système de droit fondamentalement européen ${ }^{4}$. L'histoire universelle du droit international s'est donc opérée sur le terrain colonial, si bien que l'élaboration de ses règles est intimement liée au projet de domination coloniale. Par domination coloniale, on entend l'ensemble des processus d'assujettissement et de conquête des peuples non européens par des peuples européens (ou plus largement occidentaux ${ }^{5}$ ).

2 La Cour internationale de justice, dans son arrêt rendu en 2007 dans l'affaire Diallo, définit le standard minimum de traitement des étrangers comme le «champ d'application rationae materiae de la protection diplomatique ${ }^{6} »$. Mais, selon la Cour, si la protection diplomatique s'est estompée, le standard minimum de traitement étant en constante évolution «s'est étendu par la suite pour inclure notamment les droits de l'homme internationalement garantis ${ }^{7}$ ». L'approche de la Cour ne distancie pas tant les raisonnements des commissions mixtes, qui mettaient précisément en évidence «le 
respect de l'intégrité physique de la personne, le respect des biens, l'interdiction du déni de justice ${ }^{8}$ ». Dans cet article, il ne s'agit pas pour nous d'emprunter la même voie définitionnelle que la Cour. L'idée même du standard minimum de traitement dissimule des rapports de force ou de domination, ce qui est rarement la préoccupation des juristes. Ici, nous traitons de la question de l'émergence du standard minimum de traitement des étrangers en droit international comme une production coloniale. En ce sens, il nous revient donc de situer ce standard dans la perspective du " point zéro" ", autrement dit dans le schéma de pensée qui «a permis à l'homme occidental de se représenter son savoir comme le seul à même d'atteindre l'universalité et ainsi d'écarter les connaissances non occidentales comme particularistes ${ }^{10}$ ». À notre avis, la manière occidentale de se représenter, influant nécessairement sur la production normative à l'échelle internationale, a largement contribué à une hégémonie de la colonialité. Par colonialité, il faut entendre plus que le colonialisme, c'est-à-dire un processus toujours actuel de domination et constitutif de la modernité occidentale ${ }^{11}$. Plus complexe que le colonialisme, "la colonialité consiste, elle, en l'articulation planétaire d'un système de pouvoir occidental [... et] agit précisément par le biais de dispositifs civilisationnels ${ }^{12} »$.

3 Le standard minimum de traitement peut se lire dans les œuvres des juristes positivistes comme un dispositif civilisationnel ${ }^{13}$, c'est-à-dire une norme de civilisation qu'il faut imposer dans les relations commerciales et politiques internationales. En analysant les définitions proposées par ces juristes (durant la fin du XIX et la première moitié $\mathrm{du} \mathrm{xx}^{\mathrm{e}}$ siècle $\left.^{14}\right)$, nous constatons un élément implicite de colonialité. Cet élément de colonialité réside dans le fait d'établir une approche du standard international qui ne tient compte que des pratiques des États civilisés (en l'occurrence les États occidentaux). Ainsi, la doctrine internationale, prétendant souvent être neutre, a inscrit le standard minimum de traitement au rang des catégories conceptuellescoloniales ${ }^{15}$, excluant les logiques de production normative non européennes de l'arène internationale.

4 Pour mieux comprendre cette configuration du monde global, et le processus de construction de la norme dite de civilisation qui l'accompagne, il faut remonter jusqu'au Moyen Âge. En effet, depuis cette époque, l'idée de norme européenne n'était pas écartée des dites missions civilisatrices. Certes, on ne pouvait pas parler d'un système international d'États qui édictait de règles communes, mais il existait déjà un processus de pensée favorable à une confrontation de cultures. Robert A. Williams avait si bien compris cela qu'il a déclaré :

L'Occident a cherché à imposer sa version de la vérité aux peuples non occidentaux depuis le Moyen Âge. En visant la conquête de la terre, les nations occidentales colonisatrices de l'Europe et les États colonisés qui ont été dérivés de leur expansion coloniale ont été fondés sur une idée centrale : la religion, la civilisation et les savoirs occidentaux sont supérieurs aux religions, à la civilisation et aux savoirs des peuples non occidentaux. Cette supériorité, à son tour, étant la source rédemptrice du prétendu mandat de l'Occident d'imposer sa vision de la vérité et de son instrument précieux de civilisation aux peuples non occidentaux, était également l'instrument le plus vital et le plus efficace de l'Empire pendant la conquête génocidaire et la colonisation des peuples non occidentaux du Nouveau Monde $^{16}$.

5 L'usage de la norme de civilisation pour réglementer les questions liées à l'investissement étranger à la fin du $\mathrm{XIX}^{\mathrm{e}}$ et durant la première moitié du $\mathrm{XX}^{\mathrm{e}}$ siècle ${ }^{17}$ est 
un produit, un exemple du processus très complexe de confrontation civilisationnelle dont les origines sont moyenâgeuses.

Les juristes modernes placent la civilisation occidentale au cœur de la définition de la norme internationale de traitement, et reproduisent ainsi (mais autrement) le schéma de la domination coloniale. C'est alors que s'établit une dynamic of difference, c'est-à-dire un processus continu de création du fossé entre les cultures ou les traditions, en démarquant l'une comme «universelle» et «civilisée» et l'autre comme " particulière » et «non civilisée ${ }^{18}$ ». C'est donc par le biais de cette dynamique que le droit international affirme son universalité et assure l'impérialisme continu des «États civilisés ». En conséquence, l'État « civilisé », étant celui qui s'arroge le droit de définir le «standard universel », peut se permettre de légitimer toute intervention dans les affaires internes de l'État "non civilisé », en cas de non-respect du standard minimum de traitement. Dans le cadre de cet article, notre objectif est de montrer que le standard minimum de traitement est créé au cœur de cette dynamique de la différence, qui renforce le préjugé occidental vis-à-vis des peuples non occidentaux ${ }^{19}$.

7 Selon la doctrine internationale de la fin du xIX siècle, il était quasiment normal pour les États européens de mettre en place un ensemble de dispositifs visant à combler le déficit de civilisation des peuples non civilisés. Une telle question permet d'explorer les rapports entre culture et standard de traitement et d'interroger la manière dont un type particulier de standard s'active en s'identifiant à un ensemble spécifique de pratiques culturelles (c'est-à-dire occidentales) et en excluant d'autres. Cette approche est d'autant plus importante qu'elle nous offre l'occasion de fixer le regard sur les lectures non européennes du standard européen de traitement et sur les résistances des États en développement face aux différentes formes d'ingérences légitimées par ce standard.

8 L'étude critique du standard minimum de traitement des étrangers peut révéler les contradictions inhérentes au droit international et, donc, les rapports de force qui l'animent continuellement. Notre contribution propose des pistes de réflexion sur une question maintes fois traitée - mais encore actuelle - en droit international, à savoir dans quelle mesure la question du colonialisme peut-elle se manifester - implicitement ou pas - dans la production d'une norme internationale? Ou simplement comment une norme de droit international peut-elle être révélatrice d'une véritable question coloniale ? À travers l'exemple du standard minimum de traitement des étrangers, il s'agit de montrer comment une norme peut se construire et s'imposer comme idéologie, en dissimulant le fondement colonialiste qui l'aide dans sa prétention universaliste.

9 Cet article contient deux parties. Dans la première partie, nous esquissons une généalogie coloniale du standard minimum de traitement des étrangers (1). Nous cherchons, d'abord, à démontrer comment cette norme participe de la construction d'une différence entre civilisés et non civilisés et contribue ainsi à une forme d'exclusion de certaines cultures. Ensuite, nous analysons l'idéal d'universalité qui caractérise ce standard européen (standard dit de civilisation) en mettant en évidence le sentiment de préjugé occidental qui l'accompagne dans son déploiement colonial. Au nom de ce préjugé, les cultures non occidentales sont contraintes de suivre la voie de la civilisation en modifiant leur structure normative locale. Dans la deuxième partie, nous analysons les résistances des États non occidentaux et les réformes qu'ils ont proposées. Nous prenons notamment en compte les tentatives (inspirées 
particulièrement de Calvo) d'une déconstruction décoloniale ${ }^{20}$ de ladite norme de civilisation ${ }^{21}(2)$.

\section{La colonialité du standard minimum de traitement des étrangers en droit international}

Les premières manifestations d'un droit protecteur des opérateurs étrangers remontent au XVII ${ }^{\mathrm{e}}$ siècle « avec la volonté des États européens de protéger les biens de leurs nationaux se trouvant sur un territoire étranger ${ }^{22}$ ». C'est Vattel, dans son œuvre maîtresse le Droit des gens, qui souligne que «quiconque maltraite un Citoyen [occidental] offense indirectement l'État, qui doit protéger ce Citoyen ». « Le Souverain de celui-ci doit venger son injure, obliger, s'il le peut, l'agresseur à une entière réparation, ou le punir ; puisqu'autrement le Citoyen n'obtiendrait point la grande fin de l'association Civile qui est la sûreté. » Il ajoute encore : «De même, le Souverain demande justice, ou use de représailles, non seulement pour ses propres affaires, mais encore pour celles de ses sujets, qu'il doit protéger [et] dont la Cause est celle de la Nation $^{23}$.» En réalité, ce passage reflète non seulement la volonté des États européens de protéger les biens de leurs nationaux se trouvant sur un territoire étranger, mais surtout exprime l'idée de conquête qui a caractérisé le droit international ${ }^{24}$.

11 L'idée occidentale de la conquête du monde n'est pas étrangère à l'imposition d'une conception du droit international avec ses principes fondamentaux, ses dispositifs de fonctionnement et ses institutions. L'histoire du droit international des investissements, tout comme l'histoire de ses principes et concepts, est substantiellement liée à l'expansion coloniale européenne dès le début du XVII siècle. Concept pionnier du droit international des investissements, le standard minimum de traitement est donc indissociable de l'histoire coloniale du droit international. C'est la thèse généralement défendue par les chercheurs du Third World Approaches to International Law (TWAIL) ${ }^{25}$, à savoir que le droit international n'est pas qu'un ensemble formel de règles garantissant l'égalité entre les États, mais aussi un système qui enracine l'inégalité formelle et produit la domination politique et économique des pays en développement par les pays traditionnellement exportateurs de capitaux. Dans la veine des approches tiers-mondistes et décoloniales, nous pouvons établir l'hypothèse que l'émergence du standard minimum de traitement en droit international est la preuve de la consécration des intérêts du capital au détriment des valeurs plus humaines et non essentiellement économiques. Ce déséquilibre structurel au profit du capital est le propre même d'un droit international universel dont la nature est liée à une forme d'impérialisme politique et culturel.

Dans cette première partie, nous traitons du caractère impérialiste et colonial du standard minimum de traitement (1.2). Ce qui nous oblige, avant tout, à relater l'histoire de la notion de justice civilisée, laquelle peut être considérée comme l'ancêtre de la norme internationale de traitement des investisseurs durant l'ère modernecoloniale (1.1). Né sur le terreau positiviste, le standard minimum de traitement des étrangers a adopté l'exclusion des pratiques non occidentales comme mode d'agir et a ainsi révélé l'éthique coloniale animant le droit international. 


\subsection{Les origines profondes du standard de justice civilisée}

13 Pour mieux comprendre l'idée de norme de justice civilisée en droit international des investissements et l'esprit de conquête qui en est le fondement, il faut tenir compte de sa genèse c'est-à-dire des premiers moments de l'expansion européenne. En effet, James Muldoon affirme que l'expansion européenne a commencé au Moyen Âge, particulièrement en «1095, l'année où le pape Urbain II (1088-1099) proclame la première croisade ${ }^{26}$ ». L'auteur a reconnu qu'à cette époque la " motivation religieuse » n'a jamais été l'« unique caractéristique » de l'expansion européenne, en d'autres termes «les motivations économiques et sociales étaient inextricablement associées dans une culture religieuse ${ }^{27} »$. Toutefois, du point de vue du droit international, l'année charnière dans l'histoire de la conquête européenne c'est 1245, car elle marque la « confrontation des civilisations ${ }^{28}$ » entre l'Europe et le monde non européen. C'est l'année où le Pape Innocent IV (1243-1254) commence à poser la question de l'articulation entre chrétiens et infidèles ${ }^{29}$. Incité par la domination soulevée par les croisades, Innocent IV cherche à répondre à la question suivante: "Est-il licite d'envahir une terre que possèdent les infidèles ou qui leur appartient »? En réponse à cette question, il dit ceci : « [l]es hommes peuvent choisir leurs dirigeants eux-mêmes... La souveraineté, les biens et la juridiction peuvent exister licitement, sans péché, parmi les infidèles, ainsi que les fidèles ${ }^{30}$ ». Toutefois, en tant que « vicaire du Christ », le Pape « [avait] le pouvoir non seulement sur les chrétiens, mais aussi sur les infidèles ». Et donc, «le Pape [pouvait] accorder des indulgences à ceux qui envahissent la Terre sainte dans le but de la reprendre, bien que les Sarrasins la possèdent ». James Muldoon a beaucoup insisté sur le rôle qu'a joué Innocent IV dans la question de la confrontation des civilisations. Sa discussion sur le droit naturel des infidèles de se gouverner est une étape importante dans l'évolution du droit international. Il a souvent été cité dans les débats du XVI ${ }^{\mathrm{e}}$ siècle sur les droits des habitants du Nouveau Monde face à la conquête espagnole des Amériques.

Sous l'influence évidente d'Innocent IV, Vitoria va établir le droit des gens, « qui devait devenir le droit international non seulement des pays chrétiens, mais du monde $e^{e n t i e r}{ }^{31}$ ". Selon James Brown Scott, le travail de Vitoria " attribue à la découverte de l'Amérique l'expansion du droit international jusqu'à ce qu'il soit devenu un système de règles de conduite universelle ", en d'autres termes il a annoncé l'avènement d'une « communauté internationale composée de toutes les nations [civilisées] ${ }^{32}$ ». De notre point de vue, Vitoria est finalement important pour avoir été un pionnier sur la question de la légitimité de l'impérialisme européen.

L'importance de Vitoria dans le débat sur les rencontres entre l'Europe et le Nouveau Monde est confirmée par l'affirmation selon laquelle «le droit international, tel qu'il existait à l'époque de Vitoria, ne précédait pas et ne résolvait donc pas le problème des relations hispano-indiennes »: «le droit international a plutôt été créé à partir des problèmes uniques générés par la rencontre entre les Espagnols et les Indiens ${ }^{33}$. » Nous avons là une compréhension liminaire de la confrontation des civilisations, qui aura plus tard un impact majeur sur la définition du standard européen de civilisation.

16 L'argument de Vitoria est tellement important qu'il influencera le débat sur la civilisation durant le $\mathrm{xIX}^{\mathrm{e}}$ siècle. Il sera pris en compte notamment dans la fabrication de la différence entre peuples européens et peuples non européens, entre normes européennes et normes non européennes. C'est ainsi que Vitoria va décrire une 
personnalité indienne structurée par une organisation sociale primitive ou rudimentaire. Ainsi, selon lui, toute relation internationale entre un État européen et les Indiens paraît inconcevable étant donné que les deux États ne sont pas sur un même pied d'égalité. Aux yeux de Vitoria, l'Indien a une capacité rationnelle limitée, mais cela ne lui empêche pas de comprendre le ius gentium. Il ajoute que l'Indien et l'Espagnol diffèrent considérablement dans la mesure où « les pratiques sociales et culturelles [du premier] sont en contradiction avec les pratiques requises par les normes universelles ${ }^{34} »$. Autrement dit, dans la confrontation coloniale des Espagnols face aux Indiens, ce sont des «pratiques culturelles particulières des Espagnols [qui] prennent l'apparence d'universalité parce qu'elles semblent provenir de la sphère du droit naturel ». Néanmoins, selon Antony Anghie, dans l'esprit de Vitoria, l'Indien de l'Amérique du Nord est «schizophrène, [donc il est] à la fois pareil et contraire à l'Espagnol ${ }^{35}$ ». Ce caractère schizophrénique s'explique, selon Vitoria, à partir de la reconnaissance du potentiel d'universalité chez l'Indien, mais ce "potentiel ne peut être réalisé que par l'adoption ou l'imposition des pratiques universellement applicables de l'Espagne ${ }^{36} »$. Il s'ensuit que les Espagnols bénéficiaient d'un important droit d'intervention leur permettant d'asseoir leur domination sur ces peuples non européens. De telles prémices d'une lecture anthropologique du droit international légitimant la domination de certains peuples par d'autres sont présentes chez les Lumières, parfois plus radicales ${ }^{37}$.

L'auteur de Qu'est-ce que les Lumières?, Kant, expose les motifs qui permettent de distinguer les peuples :

Dans les pays chauds, les hommes mûrissent plus vite à tous égards, mais ils n'atteignent pas la perfection des zones tempérées. L'humanité atteint la plus grande perfection dans la race des Blancs. Les Indiens jaunes ont déjà moins de talent. Les Nègres sont situés bien plus bas ${ }^{38}$.

Pour démontrer encore plus l'impératif civilisationnel de la domination occidentale et blanche dans l'œuvre des Lumières, on peut poursuivre avec Kant :

En considérant les nations non civilisées, on voit bien, si longtemps qu'elles restent au service des Européens, qu'elles ne peuvent s'habituer à leur manière de vivre. Ce n'est pas chez elles [...] un noble penchant à la liberté ; ce n'est qu'une certaine rudesse, puisque d'une certaine manière l'animal n'a pas encore développé en soi l'humanitée ${ }^{39}$.

19 Les affirmations de Kant, comme celles qui les précèdent, ont une importance considérable, car elles postulent clairement le préjugé colonial qui va animer le droit international. Nous avons là des idées qui placent le droit international sur le terrain de la pensée eurocentrée ${ }^{40}$. La pensée eurocentrée est, ainsi, celle qui fixe les conditions d'adhésion à la famille des nations civilisées. Une telle adhésion ne peut se réaliser que par le respect du standard de civilisation, celui-ci étant un cadre minimum que tous les États voulant revendiquer l'appartenance à la grande famille des nations sont tenus de reconnaître. Ainsi, l'État non occidental qui souhaite satisfaire aux exigences du droit international doit accorder aux étrangers un traitement correspondant aux mesures requises par les États civilisés. Les puissances dites civilisées étendaient leur domination «sur les États situés hors des limites de l'Europe à mesure qu'ils s'adaptaient à la civilisation européenne $\mathrm{e}^{41} »$.

Les idées du pape Innocent IV, de celles de Vitoria ou de celles des Lumières traduisent les premières formulations d'une norme européenne de civilisation qui deviendra très à la mode à partir de la fin du XIX siècle. Les positivistes du droit international 
présenteront plus tard les pratiques européennes comme s'il s'agissait purement et simplement de «normes universellement applicables auxquelles les peuples colonisés doivent se conformer s'ils veulent éviter les sanctions, et devenir membres à part entière [de la communauté internationale ${ }^{42}{ }^{4}$. Ainsi s'établit peu à peu, en droit international, un processus continu de fabrication de la distinction, c'est-à-dire une tendance qui définit les cultures non européennes comme inadaptées aux pratiques sociales civilisatrices. En d'autres termes, toute culture qui n'est pas capable de faire l'effort de se conformer au système d'organisation politico-juridique dont le principe est ladite norme universelle sera réputée barbare, non civilisée ou simplement inférieure.

21 La hiérarchisation faite par Kant sera reprise par le célèbre publiciste James Lorimer. Ce dernier a présenté éloquemment une distinction suprême au sein de l'espèce humaine, qui va influencer pendant longtemps la jurisprudence internationale ${ }^{43}$. Influencé par Montesquieu ainsi que des anthropologues et ethnologues tels que Lewis Henry Morgan et Arthur de Gobineau, James Lorimer précise : «phénomène politique, l'humanité, dans son état actuel, se divise en trois zones ou sphères concentriques: celle de l'humanité civilisée, celle de l'humanité barbare et celle de l'humanité sauvage ${ }^{44}$.» Comme l'a cru aussi Vitoria, James Lorimer pense que "[s]avages are incapable of municipal organisation beyond its most rudimentary stages; and yet it is by means of municipal organisation that men cease to be savages ». Et, s'agissant des États barbares, il s'appuie sur Grotius pour affirmer que ces derniers «n'ont jamais été reconnus par les nations européennes; et [que] la conquête de l'Algérie par la France n'a pas été considérée comme une violation du droit international ».

En conséquence, conformément à cette distinction fondamentale, la forme d'organisation sociopolitique ou de gouvernement d'un État non européen est devenue centrale pour déterminer si celui-ci était acceptable parmi les États civilisés. Qu'il s'agisse de Wheaton, de Westlake ou encore de Hall, ils admettent tous que l'obtention du statut d'État civilisé exige la conformité préalable aux standards européens ${ }^{45}$.

Compte tenu de ce qui a été avancé, nous pouvons dire que l'histoire du droit international s'inscrit dans un continuum complexe de rapports de force. Ces rapports de domination ont pris des formes variées au cours des époques: fidèle/infidèle, sauvage/civilisé ou barbare/civilisé, moderne/non-moderne, développé/sousdéveloppé. En conclusion, la définition du standard de civilisation en droit international est la résultante d'une longue histoire de confrontation de civilisations laissant entrevoir un lent déploiement de l'éthique impérialiste occidentale.

\subsection{Le standard minimum de traitement ou l'exemple fidèle d'une norme de civilisation : point de rencontre colonial entre l'Occident et «les autres"}

Durant le $\mathrm{XIX}^{\mathrm{e}}$ et le début du $\mathrm{xx}^{\mathrm{e}}$ siècle, le discours juridique sur le standard minimum de traitement des investisseurs étrangers en droit international a épousé la logique de la norme de civilisation. Par standard minimum de traitement, il faut entendre avant tout une norme occidentale de protection internationale de l'étranger que les États hôtes étaient tenus d'appliquer. Nous présentons ici cette norme comme un point de rencontre coloniale, puisque les États occidentaux (anciennement colonisateurs) peuvent effectuer par le biais de ce dispositif civilisationnel une intrusion dans les 
affaires internes des États anciennement colonisés. Le standard minimum de traitement se fera remarquer tant sur le terrain de la doctrine que sur celui de la jurisprudence internationale.

Tout d'abord, l'élaboration doctrinale du standard minimum de traitement s'inscrit dans une logique impérialiste du droit international. Les juristes du droit international vont se servir du positivisme pour légitimer l'impérialisme des États occidentaux ${ }^{46}$. En présentant la discipline juridique selon les fondements positivistes, c'est-à-dire comme ayant un caractère absolument scientifique et universel, les juristes sont parvenus à dissimuler le mode d'être colonial des principes juridiques dits universels.

En outre, la définition du standard minimum de traitement s'est reposée sur une fameuse distinction entre États civilisés et États non civilisés, un des aspects fondamentaux $\mathrm{du}$ positivisme juridique naissant au $\mathrm{XIX}^{\mathrm{e}}$ siècle $^{47}$ qui a ravivé le sentiment de préjugé colonial. Il est clair que pour des juristes tels que Henry Wheaton, le droit international relevait $\mathrm{du}$ domaine de compétence exclusive des sociétés occidentales ou civilisées ${ }^{48}$ et non de la compétence des sociétés non civilisées. C'est, en fait, le prolongement de la pensée du célèbre juriste néerlandais, Cornelius Van Bynkershoek, qui avait déjà soutenu au XVIII ${ }^{\mathrm{e}}$ siècle que « seule la pratique des États civilisés acquiert la valeur juridique ${ }^{49}$ ». Il ajoutait précisément : «le droit des gens est [une relation qui s'établit] en vertu de la lumière de la raison, entre des peuples sinon entre tous, du moins certainement la plus grande partie, et les plus civilisés ». Dans la même veine, $\mathrm{H}$. Wheaton va affirmer en 1866 : «Il n'en est certainement pas de même pour toutes les nations et tous les États du monde. Le droit public, à quelques petites exceptions près, a toujours été et est toujours limité aux peuples civilisés et chrétiens de l'Europe ou à ceux d'origine européenne ${ }^{50}$. " Le juriste et diplomate américain a clairement établi un fossé entre le monde européen et le monde non européen. Une telle distinction n'est pas du tout anodine puisqu'elle affirme l'imposition du droit international européen sur les sociétés non européennes dites non civilisées, d'où ce que nous appelons un dispositif de colonialité.

Mais, pour mettre plus encore en lumière l'aspect colonial et impérialiste du standard minimum de traitement des investisseurs étrangers en droit international (dont les racines plongent dans la norme dite de civilisation), il faut revenir au discours célèbre de Lord Palmerston, homme d'État britannique.

Certes les prémices d'un standard de civilisation remontent à des temps très anciens, mais c'est dans le discours de Lord Palmerston, prononcé le 25 juin 1850 à la Chambre des communes à la suite de l'affaire Don Pacifico ${ }^{51}$, que l'on va trouver l'une des premières justifications officielles du standard minimum de traitement des étrangers. Selon lui, l'exigence du respect du standard international de traitement s'explique par le fait que dans certains cas : " aucune confiance ne peut être placée dans les tribunaux, ces tribunaux étant de par leur composition et leur nature, non susceptibles de susciter l'espoir d'obtenir justice de leur part ${ }^{52}$. » Il poursuit en soulignant que, puisque «les gouvernements sont arbitraires ou despotiques, car là-bas les tribunaux sont sous le contrôle du gouvernement et que justice ne peut être rendue ", l'intervention armée ou non est donc légitime. On peut comprendre que Lord Palmerston défend ici l'idée d'intervention (par les moyens diplomatiques ou le recours à la force armée) dans les affaires internes d'un État hôte en l'absence de garanties juridiques réelles ou d'impartialité des tribunaux. Mais ce qui nous intéresse le plus dans son discours c'est le fait, pour justifier sa position, de se référer à «the maintenance of peace, [...] the 
advancement of civilization [...] the welfare and hapiness of mankind ", autrement dit des formules qui participent largement de la définition de la mission du droit international européen ${ }^{53}$.

Dans les propos de Lord Palmerston, on peut voir clairement l'intention de combler le vide de civilisation dans les sociétés non occidentales. Mais, dans les affirmations d'Elihu Root (1845-1937), président de la jeune American Society of International Law, on va retrouver une version beaucoup plus idéologique des principes évoqués par l'homme politique britannique. C'est ainsi qu'il décrit en termes clairs la position des pays exportateurs de capitaux :

chaque pays est tenu d'accorder aux ressortissants d'un autre pays de son territoire le bénéfice des mêmes lois, de la même administration, de la même protection et de la même compensation pour le préjudice qu'il cause aux citoyens de son propre pays, et ce ni plus ni moins : à condition que la protection que le pays accorde à ses propres citoyens soit conforme à la norme établie de la civilisation. Il existe une norme de justice très simple, très fondamentale et acceptée de manière aussi générale par tous les pays civilisés qui fait partie du droit international. Un pays est en droit de mesurer le niveau de justice dû à un étranger par la justice qu'il accorde à ses propres citoyens que lorsque son système de droit et d'administration est conforme à ce critère général. Si le système juridique et administratif d'un pays n'est pas conforme à cette norme de justice, bien que la population du pays puisse être contente ou contrainte de vivre sous ce régime, aucun autre pays ne peut être contraint de l'accepter comme offrant une mesure de traitement satisfaisante à ses citoyens ${ }^{54}$.

Il y a dans la déclaration d'E. Root l'idée que le traitement national est ce que les étrangers ont le droit d'espérer, mais il n'est satisfaisant que si l'ordre juridique local comporte un ensemble de normes substantielles et procédurales conformes à des règles minimales de droit international. C'est ainsi que le droit européen des nations civilisées de tradition judéo-chrétienne a été établi comme système normatif applicable pour déterminer si une nation était civilisée ou non, et donc si elle était recevable ou non dans la société internationale. Finalement, sa recevabilité ou son exclusion du droit international dépendait de la question de savoir si son organisation politique reflétait les normes sociopolitiques européennes.

31 Edwin Borchard, citant Haufmann, a écrit que le droit international a établi l'existence d'un standard international auquel tous les États doivent se conformer sous peine d'engager leur responsabilité. Ce principe a été consacré par la Cour permanente de justice internationale qui « a jugé que les étrangers ont droit à un traitement supérieur à celui des nationaux chaque fois qu'ils sont traités contrairement au droit international coutumier ${ }^{55}$ ». E. Borchard précise: "[1]orsque le droit interne concernant les étrangers se trouve en deçà des exigences de la norme internationale, notamment en cas de déni de justice, l'étranger a même un droit à un traitement supérieur à celui que le droit interne accorde aux ressortissants. » En conséquence, les exigences du droit international sont satisfaites lorsque l'égalité de traitement accordée par l'État hôte aux nationaux et étrangers correspond aux mesures requises par le droit international. Le standard du droit international auquel est soumis le traitement des étrangers étant reconnu par les États civilisés, sa violation «peut donner lieu à une action diplomatique des gouvernements ».

Ce point de vue était celui d'une doctrine majoritaire au XIX siècle en Europe, voire aux États-Unis ${ }^{56}$. En effet, la plupart des publicistes se sont mis d'accord sur l'idée qu'en droit international un État doit offrir aux étrangers une protection judiciaire fidèle aux 
principes internationaux. C'est ainsi que le juriste italien Dionisio Anzilotti a exprimé la même préoccupation en évoquant l'hypothèse d'une justice qui fait défaut lors d'un procès impliquant un étranger. $\mathrm{Si}$, pour lui, « les États accomplissent leur obligation dès qu'ils ont octroyé aux étrangers le recours à la justice [...], il n'en reste pas moins vrai qu'il est des hypothèses - ainsi que l'expérience le prouve - dans lesquelles, malgré les apparences, la protection judiciaire fait en réalité défaut, ce qui équivaut au déni de justice ${ }^{57}$ ». C'est à peu près la même défense du standard minimum de traitement que l'on retrouve dans les écrits de Lapradelle et Politis : «[d]ire que l'étranger ne saurait être mieux traité que le national [...] est une formule inexacte ${ }^{58}$.» Ainsi, le national et l'étranger étant de statut juridique différent, ils ne peuvent aucunement faire l'objet d'un même traitement. En d'autres termes, le traitement du national est déterminé par le droit interne et celui de l'étranger par le droit international; le second est susceptible d'être sur certains points plus étendu que le contenu des règles du premier. Commentant l'enracinement du standard de civilisation dans le droit international, le juriste Georg Schwarzenberger a très bien résumé la situation :

le critère qui permet de savoir si un État est civilisé et a donc droit à être pleinement reconnu en tant que personnalité internationale s'applique généralement à la question de savoir si son gouvernement est suffisamment stable pour prendre des engagements contraignants en vertu du droit international et s'il est capable et disposé à protéger de manière adéquate la vie, la liberté et la propriété des étrangers ${ }^{59}$.

Puisque les étrangers en question étaient quasiment tous des citoyens issus des États civilisés, c'est-à-dire des pays du Nord, dans la pratique cela signifiait que tous les peuples ne partageant pas les lois et les coutumes occidentales étaient automatiquement exclus de la société internationale.

Bref, en affirmant que seule la pratique des États européens est déterminante pour créer un droit international civilisateur, comme le veut l'article 38 du statut de Cour permanente internationale de justice, l'idéologie occidentale dominante a exclu les pratiques des États non européens du domaine du droit et les a privés de leur adhésion de faire valoir des principes juridiques reconnus comme légaux. Ayant fondé le standard positiviste de traitement des étrangers sur la fameuse distinction entre civilisés et non civilisé, les juristes internationalistes ont présenté au grand jour une nouvelle éthique coloniale garantissant de nouvelles formes d'intrusion dans les territoires non occidentaux.

Un tel sentiment de préjugé colonial est également présent dans la jurisprudence internationale. D'ailleurs, bien avant que l'article 38 du statut de la Cour permanente internationale de justice «[...] a fait des principes généraux de droit reconnus par les États civilisés une source de droit international coutumier, les services des affaires étrangères et les tribunaux arbitraux se sont appuyés sur ces principes généraux pour élaborer un standard souple [standard minimum] qu'ils appliquaient constamment dans les pratiques entre États ${ }^{60} »$. Par exemple dans l'affaire Dr Baldwin's Minatitlan Claims ayant opposé les États-Unis et le Mexique, la commission des réclamations a déclaré :

[s]i le Mexique souhaite maintenir son rang et son amitié parmi les nations civilisées de la terre, il doit mettre ses lois sur le même pied que les autres nations en ce qui concerne les relations avec les étrangers. L'oppression exercée à l'encontre de ses citoyens est une chose; la pratique d'une oppression semblable 
sur des étrangers en est une autre. Ces derniers ont le droit de faire appel à la protection diplomatique en cas de préjudice ${ }^{61}$.

Un peu plus tard, durant la première moitié du $\mathrm{xx}^{\mathrm{e}}$ siècle, le même argument de la commission dans l'affaire Baldwin sera reproduit dans l'affaire Hopkins (ayant encore une fois opposé les États-Unis et le Mexique en 1923) : le tribunal conclut que, en vertu de leur recours diplomatique et arbitral, les étrangers pouvaient parfois recevoir un traitement plus large et plus libéral que celui accordé aux nationaux. Les juges ont ajouté que les avantages octroyés aux étrangers ne peuvent aucunement signifier une discrimination à l'encontre des citoyens de l'État hôte ${ }^{62}$.

En 1926, le tribunal dans l'affaire Roberts a encore confirmé la «pertinence » du standard de civilisation :

Roberts a bénéficié du même traitement que celui accordé à toutes les autres personnes [les citoyens locaux] [...] Les faits concernant l'égalité de traitement des étrangers et des nationaux peuvent être importants pour déterminer le bien-fondé d'une plainte pour mauvais traitement d'un étranger. Mais cette égalité n'est pas le test ultime de la pertinence des actes des autorités à la lumière du droit international. En gros, le test ultime consiste à déterminer si les étrangers sont traités conformément aux standards ordinaires de civilisation ${ }^{63}$.

On peut en déduire que le standard de civilisation, en tant que norme européenne, légitime l'intrusion coloniale ${ }^{64}$ en établissant la seule condition pour qu'un État non européen puisse être considéré comme civilisé : fournir à l'étranger européen le même traitement qu'il s'attendrait à recevoir en Europe sous peine d'interventions diplomatiques ou armées. Dans cette perspective, le standard minimum de traitement international en tant que norme de civilisation est un prolongement de l'état des rapports de force entre États anciennement colonisateurs et États anciennement colonisés. Puisque dans le contexte des $\mathrm{XIX}^{\mathrm{e}}$ et $\mathrm{xx}^{\mathrm{e}}$ siècles la promotion des intérêts commerciaux dans les États non occidentaux s'accompagnait du risque d'intervention militaire de la part des États occidentaux, la seule possibilité pour ne pas tomber sous le coup d'une telle ingérence était d'intégrer les différentes pratiques et politiques européennes dans les modes de vie locaux.

John Westlake (1828-1913), célèbre professeur et ancien président de l'Institut de droit international, a résumé en quelques mots le test rudimentaire de civilisation: "[1]orsque des personnes de race européenne entrent en contact avec des tribus américaines ou africaines, la nécessité première est de mettre en place un gouvernement sous la protection duquel les premiers peuvent mener la vie complexe à laquelle ils ont été accoutumés chez eux ${ }^{65}$.» Il n'affirme pas que les pays non occidentaux sont incapables de civilisation, mais que cela ne peut se faire que par une transformation des lois locales selon les exigences des États occidentaux. Les systèmes locaux de justice étant inadéquats pour les États européens, il n'est pas question de soumettre un citoyen occidental à ces systèmes.

41 Cela impliquait une double exigence pour les États non occidentaux. Premièrement, ils devaient faire preuve de respect à l'égard de la "société internationale ", afin de maintenir les liens diplomatiques nécessaires et de préserver leurs relations avec les États européens. Deuxièmement, ils étaient tenus de réformer leurs systèmes juridiques et politiques en tenant compte substantiellement des normes européennes dans leur ensemble et d'abandonner leur propre potentiel normatif, du moins dans leurs rapports avec les Occidentaux présents sur leur territoire. 

américains et caribéens qui ont compris que les normes occidentales dites de civilisation n'avaient aucune mission de pacifier et de promouvoir une relation harmonieuse entre les États, mais au contraire servait de prétexte pour justifier des interventions dans les affaires intérieures des États nouvellement indépendants. Ainsi, Carlos Calvo, ayant commenté plusieurs interventions des États européens en Amérique latine et dans les Caraïbes, a observé qu'«[à] côté de mobiles politiques, les interventions ont presque toujours eu comme prétexte apparent des lésions d'intérêts privés, des réclamations et des demandes d'indemnités pécuniaires en faveur de sujets ou même d'étrangers dont la protection n'était la plupart du temps nullement justifiée en droit strict ${ }^{66} »$. Selon lui, l'intervention des puissances européennes dans les affaires des divers États du Nouveau Monde, par ses mobiles comme par ses résultats, ne reposait très souvent sur aucun fondement légitime, mais de préférence reproduisait une simple logique coloniale ${ }^{67}$. occidentales, a écarté tout élément qui pourrait paraître spécifique à un État non occidental. L'idéal d'universalité qui caractérise le standard de civilisation s'est complètement réalisé par la suppression substantielle (processus historiquement assez récent) du monde non occidental. Autrement dit, au cours de ce processus le concept de civilisation a été utilisé comme une logique d'exclusion des valeurs autres, c'est-à-dire celles qui s'éloigneraient du cadre de l'identité non occidentale ${ }^{68}$. Ce faisant, l'élaboration du standard de traitement international s'est appuyée sur un dispositif civilisationnel-colonial aidant à légitimer une certaine "épistémologie juridique universelle » aux dépens des « singularités épistémiques ».

Considérant que le standard minimum de traitement tel qu'il a été jadis défini n'est plus aujourd'hui à la mode, cette discussion peut paraitre sans grande importance pour certains. En revanche, la question ici soulevée ne peut pas être facilement abandonnée dans les poubelles du passé puisqu'elle est encore d'une certaine actualité. D'ailleurs, les configurations politico-économique, juridique et géopolitique mises en place depuis la rencontre coloniale, et l'ensemble des mythes, récits et métaphores qui les accompagnent, continuent de décrire la vie du droit international et définissent l'ère globale actuelle ${ }^{69}$. Par exemple, les couples binaires tels que moderne/non moderne, civilisé/non civilisé, développé/sous-développé ont façonné une éthique européenne qui reste vivante dans le droit international contemporain ${ }^{70}$. Selon Martti Koskenniemi, l'ensemble de ces couples conceptuels qui sont au cœur du droit international indiquent clairement les expériences et conceptualisations européennes et permettent à l'Europe de régner encore aujourd'hui en tant que "référent silencieux de la connaissance historique ${ }^{71} »$. Enfin, un exemple concret qui illustre cette idée est que les normes substantielles et procédurales de protection des investisseurs étrangers, telles qu'elles sont aujourd'hui définies, sont le fruit du standard international de traitement.

À travers cette modeste discussion sur la colonialité du standard minimum de traitement des étrangers, nous arrivons à deux conclusions: d'une part, la notion occidentale de civilisation qui a largement contribué à définir le standard minimum de traitement a permis à l'occident de réaffirmer sa domination sur le reste du monde ; d'autre part, la fabrication idéologique de ce standard a fait de lui un exemple parmi tant d'autres de reproduction des inégalités normatives et d'exclusion à l'échelle mondiale ${ }^{72}$. De ce fait, l'obligation d'inclure le standard minimum de traitement dans 
les ordres juridiques internes s'inscrit dans un long processus de pensée prônant l'intégration impérative de la civilisation occidentale. C'est pourquoi nous avons soutenu que le standard minimum de traitement a réaffirmé une forme de rencontre coloniale $^{73}$. Il nous reste alors à démontrer comment cette opération idéologique occidentale a été contestée et a du coup occasionné l'affirmation de nouvelles réponses en droit international.

\section{Les tentatives de déconstruction du standard minimum de traitement des étrangers en droit international}

Si le référencement occidental s'avère dominant dans le jeu des relations internationales, il faut bien admettre que le droit international demeure un champ de théories rivales, de rapports de force et d'intérêts divergents. Pour preuve, il n'a pas été facile d'imposer le standard minimum de traitement aux peuples et territoires non occidentaux. Ces derniers, les États d'Amérique latine en particulier, ont cherché à établir leur propre standard de traitement de l'étranger et à affirmer une approche de la souveraineté pleine et entière.

Pour soutenir leurs États, certains juristes du tiers-monde ne se sont pas contentés de reproduire un droit international comme s'il était complètement formé et prêt à être appliqué. Au contraire, ils ont dénoncé un processus d'assimilation des sociétés au sein d'un système "eurocentrique " stable. Ainsi, malgré la confrontation coloniale au $\mathrm{XIX}^{\mathrm{e}}$ siècle et au début $\mathrm{du} \mathrm{xx}^{\mathrm{e}}$ siècle qui a opposé les pays occidentaux «absolument souverains » et les sociétés non européennes qualifiées de "souverainement limitées ", il y a eu l'expression d'autres histoires du droit international comme ce fut le cas dans les œuvres de Calvo et de Bello.

L'attitude des États et juristes latino-américains doit être perçue à la fois comme tentative de déconstruction de la colonialité et tentative de construction de la décolonialité. Il ne s'agit pas de deux moments séparés, mais de deux faces d'une même monnaie. En d'autres termes, il y a, d'une part, une volonté tiers-mondiste de contrecarrer tout projet colonial dissimulé sous un voile normatif obscur et, d'autre part, cette volonté est consacrée par une législation qui s'oppose au dit universeloccidental.

Dans les œuvres de certains penseurs de l'Amérique latine, on peut trouver les schèmes d'une doctrine juridique hostile à l'égard de toute forme nouvelle d'intrusion coloniale. Mais plus encore, cette théorie entièrement souverainiste postule un standard de traitement d'égalité entre investisseurs étrangers et nationaux. Cela dit, la surprotection libérale de l'étranger est absolument écartée par le cadre normatif de l'État hôte de l'opérateur économique étranger (2.1).

Vers la seconde moitié du $\mathrm{xx}^{\mathrm{e}}$ siècle, cette épistémologie juridique de la résistance a été poussée jusqu'à ses extrêmes limites par les États communistes et ceux du tiers-monde. C'est au forum de l'Assemblée générale des Nations unies que ce combat a été mené. On retient de cette vague nouvelle le rejet des principes classiques du droit international (dont le standard minimum de traitement) ou, sinon, leur réinterprétation radicale ${ }^{74}$ (2.2). 


\subsection{La proposition d'un standard « autre » chez les pays non occidentaux : construire pour déconstruire}

Dans son opinion individuelle émise dans l'affaire Barcelona Traction, le juge L. P. Nervo a noté que «l'histoire de la responsabilité des États en matière de traitement des étrangers est une suite d'abus, d'ingérences illégales dans l'ordre interne des États faibles, de réclamations injustifiées, de menaces et même d'agressions militaires sous le couvert de l'exercice de droit des protections, et de sanctions imposées en vue d'obliger un gouvernement à faire les réparations demandées ${ }^{75}$.»Ce commentaire nous décrit implicitement l'attitude coloniale des États occidentaux à l'égard des États de l'Amérique latine telle qu'elle s'est manifestée tout le long du xIX et même au $\mathrm{xx}^{\mathrm{e}}$ siècle. Il est vrai que ce comportement n'était pas contraire au droit international, mais révélait sa vraie nature. Par ailleurs, «s'agissant des États [non occidentaux], en particulier ceux d'Amérique latine, qui étaient la cible des interventions armées, il n'est pas exagéré de dire que ces multiples interventions armées, cette diplomatie de la canonnière comme elle devait être appelée, ont créé un véritable traumatisme historique $^{76}$ » dont les schèmes sont encore aujourd'hui présents dans la compréhension latino-américaine et caribéenne $\mathrm{du}$ droit international des investissements et de l'arbitrage transnational. Nous retenons que les défauts et lacunes du standard « universel-occidental » de protection des investisseurs étrangers ont engendré les résistances des territoires «autres» et l'affirmation d'une éthique normative singulière. En ce sens, la doctrine Calvo, qui va beaucoup influencer le standard latino-américain de protection des investisseurs étrangers, peut se lire comme l'expression juridique d'une singularité épistémique. Dans cette même veine, Frank G. Dawson et Ivan L. Head ont noté que «Perhaps it was at this point, with the formulation of the Calvo Doctrine, that the Latin American nations began to assume a distinctive, collective identity as a special legal system or suborder, with legal institutions within, yet in some aspects apart from, the Europeanized international law propounded by legal theorists in capital-exporting nations ${ }^{77}$. "

De la doctrine Calvo va naître une clause Calvo, insérée dans de nombreux contrats et dans les Constitutions par les pays latino-américains et caribéens. Selon cette clause, les investisseurs étrangers bénéficiaient du même traitement que les nationaux et n'avaient pas le droit d'impliquer leurs États d'origine dans les éventuels contentieux, en ce sens que seuls les tribunaux des États d'accueil de l'investissement étaient compétents $^{78}$.

S'inscrivant dans l'approche de Luis M. Drago, la clause Calvo voulait, en limitant le standard occidental de protection des étrangers et le principe d'intervention, empêcher une éventuelle "ruine des nations les plus faibles et l'absorption d'un gouvernement, avec toutes les facultés qui lui sont inhérentes, par les puissants de la terre ${ }^{79}$ ».

54 Chez les juristes latino-américains, les principes tels que la défense de la souveraineté nationale, l'égalité des États et l'exclusivité de la compétence territoriale ont été radicalement formulés dès la fin $\mathrm{du} \mathrm{XIX}^{\mathrm{e}}$ siècle ${ }^{80}$. Les États de la région ont ainsi voulu marquer le droit international de leur empreinte hostile. C'est pour cela que la plupart d'entre eux se dotèrent de dispositions constitutionnelles ou législatives qui consacraient ces principes ${ }^{81}$. 

ne s'est malheureusement pas imposée en droit international. La doctrine Calvo n'a donc pas été placée au rang de "principe universel» de civilisation. Au contraire, la Cour permanente de justice internationale dans son arrêt sur les concessions Mavrommatis en Palestine va confirmer la toute-puissance de la protection diplomatique et, en conséquence, la défaite de la doctrine Calvo, en soulignant que « [c]'est un principe élémentaire du droit international que celui qui autorise l'État à protéger ses nationaux lésés par des actes contraires au droit international commis par un autre État, dont ils n'ont pas pu obtenir satisfaction par les voies ordinaires ${ }^{82}$. »

Si la doctrine Calvo n'a pas été acceptée comme règle universelle, elle a toutefois été exprimée dans un standard qui postule l'égalité de traitement entre nationaux et agents économiques étrangers. Des États tels que le Chili (1833), la Fédération d'Amérique centrale (1835), le Pérou (1839), le Costa Rica (1844), l'Argentine (1857) ont adopté des Constitutions qui favorisaient le principe du traitement national. Les textes constitutionnels offraient donc une égalité de traitement quasi complète ou stipulaient un ensemble de droits que les étrangers pouvaient partager avec les nationaux ${ }^{83}$.

l'écrivait Andres Bello en $1886^{84}$, l'étranger qui entre sur le territoire de l'État d'accueil accepte sans condition ses lois et ses tribunaux et ne devait pas faire appel à son État national ou à une juridiction internationale. C'est ainsi que, dans l'arbitrage Rosa Gelbtrunk c. El Salvador, l'arbitre sir Henri Strong déclara dans son opinion individuelle que l'État national d'un étranger " has no right claim for him as against the nation in which he is resident any other or different treatment in case of loss by war-either foreign or civil-revolution, insurrection, or other internal disturbance caused by organized military force or by soldiers, than that which the latter country metes out to its own subjects or citizens. This I conceive to be now the well-established doctrine of international law ${ }^{85}$. " À travers cette opinion, sir Henri Strong prépara le chemin d'une doctrine tiersmondiste. Cette position pourtant réfutée par les États développés et leurs juristes sera plus tard reproduite avec force et complétée par les États en voie de développement lors des revendications d'un nouvel ordre international juridique et économique au début de la seconde moitié du XIX ${ }^{e}$ siècle.

\subsection{L'hypothèse d'un nouvel ordre économique international, un pas décisif vers la déconstruction des principes classiques du droit international}

$\mathrm{Au}$ milieu du xxe siècle, il y a eu une lutte radicale pour l'instauration d'un nouvel ordre économique international. Au sein des organisations internationales, les pays du tiersmonde et les États communistes allaient grouper leur action en vue de remettre en cause les grands principes du droit international. Certes, ces principes ont déjà été critiqués par certains juristes et hommes politiques d'Amérique latine, mais la doctrine critique internationale demeurait minoritaire sur le plan international. L'affirmation d'un nouvel ordre économique international va permettre une remise en cause, sinon une déconstruction fondamentale des principes classiques du droit international.

59 C'est au forum de l'assemblée générale des Nations unies que les États tiers-mondistes et communistes, en majorité, allaient défendre la souveraineté permanente sur leurs ressources naturelles. Il s'agissait pour eux « de récupérer leurs richesses naturelles, surtout minières et pétrolières, qui étaient dans les mains des entreprises occidentales

Les Annales de droit, 14 | 2020 
et qui étaient considérées comme les instruments nécessaires et, pensaient certains, suffisants du développement économique ${ }^{86}$ ». C'était également l'occasion pour ces États de donner une nouvelle orientation à un droit international dont la production théorique et politique était quasiment du domaine des États occidentaux, puisqu'« un petit syndicat d'États [...] depuis plusieurs siècles, projette "son" droit dominant sur la scène internationale en l'imposant comme "le" droit international régissant la planète tout entière ${ }^{87} \%$.

Ici, on n'aura pas la place pour traiter de tous les aspects de cette vaste lutte menée sur le plan international contre les États exportateurs de capitaux, on ne peut donc que l'effleurer. L'analyse portera sur un seul document: la résolution 3281 (XXIX) du 12 décembre 1974 de l'assemblée générale des Nations unies, qui a abouti à la Charte des droits et devoirs économiques des États. Cette Charte constitue en elle-même une puissante réaction au standard minimum de traitement. Dans la charte, l'article 2 a établi ceci :

2. Chaque État a le droit

a) De réglementer les investissements étrangers dans les limites de sa juridiction nationale et d'exercer sur eux son autorité en conformité avec ses lois et règlements et conformément à ses priorités et objectifs nationaux. Aucun État ne sera contraint d'accorder un traitement privilégié à des investisseurs étrangers; $[\ldots]$

c) De nationaliser, d'exproprier, ou de transférer la propriété des biens étrangers, auquel cas il devrait verser une indemnité adéquate, compte tenu de ses lois et règlements et de toutes les circonstances qu'il juge pertinentes. Dans tous les cas où la question de l'indemnisation donne lieu à un différend, celui-ci sera réglé conformément à la législation interne de l'État qui prend des mesures de nationalisation et par les tribunaux de cet État, à moins que tous les intéressés ne conviennent librement de rechercher d'autres moyens pacifiques sur la base de l'égalité souveraine des États et conformément au principe de libre choix des moyens.

61 La résolution proclamant la Charte des droits et devoirs économiques des États fut adoptée par 118 voix pour, 6 contre et 10 abstentions. Il s'agissait d'une victoire tiersmondiste puisque, comme l'a précisé l'arbitre dans la sentence Texaco, les pays exportateurs de capitaux ont tous voté contre ou se sont abstenus ${ }^{88}$. Cette résolution est historiquement importante dans l'histoire du droit international, car il en résultait une remise en cause de quelques principes classiques fondamentaux. Par exemple, s'agissant de la réglementation des investissements et du paiement d'une indemnité en cas de nationalisation seul le droit local était compétent, selon ladite Charte.

La Charte des droits et devoirs économiques des États est également perçue comme une victoire, ou une consécration internationale de la doctrine Calvo. Tout d'abord, selon la perspective de la Charte l'investisseur devrait être traité selon les normes locales de l'État d'accueil. En ce sens, en cas de nationalisation il ne peut être mieux et plus indemnisé que les nationaux. Ainsi, l'investisseur ne peut espérer autre chose qu'un égal traitement au national et jamais un traitement supérieur. On peut donc constater un retour de la doctrine Calvo d'autant que la non-ingérence a été clairement réaffirmée: "chaque État détient et exerce librement une souveraineté entière et permanente sur toutes ses richesses ${ }^{89} »$ et seul lui peut trancher d'éventuels litiges entre l'investisseur étranger et lui. 

en matière de règlement des différends des investissements; ce qui s'oppose complètement au standard de traitement occidental. Dans l'esprit de la Charte, aucune juridiction internationale ne doit remettre en cause la souveraineté permanente des États sur leurs ressources naturelles. On peut comprendre que la quête d'un nouvel ordre économique international repose entièrement sur l'affirmation d'une idée radicale de souverainetés ${ }^{0}$. Dans cette perspective, les activités des investisseurs devraient être soumises, en cas de litiges, à l'appréciation souveraine des juridictions nationales.

En conclusion, la Charte des droits et devoirs économiques des États a favorisé l'affirmation, sur la scène internationale, des possibilités et des principes autres que ceux traditionnellement connus par les États dits civilisés. Elle a permis une remise en cause de l'approche classique du standard minimum de traitement fondée sur une conception quasi européenne du droit international. Ainsi, elle a fait resurgir sur le terrain du droit international les grands principes de souveraineté, d'indépendance et d'égalité qui guidaient les œuvres critiques de certains juristes latino-américains, dont précisément celle de Carlos Calvo.

Cependant, si l'ensemble des résolutions de l'Assemblée générale des Nations unies ont occasionné une vive incertitude quant au contenu exact du droit positif international (concernant les questions de nationalisation et de réparation des investisseurs étrangers), elles n'ont pas propulsé les pays du tiers-monde vers le développement économique. La conséquence de la philosophie du nouvel ordre économique international « fut un détournement massif des flux d'investissements qui délaissèrent les pays du tiers-monde pour se diriger vers les pays développés ${ }^{91} »$. Le nouvel ordre économique international n'a pas pu voir le jour même s'il faut reconnaître que cette nouvelle vague tiers-mondiste a eu des répercussions sur les différentes approches critiques postcoloniales et décoloniales qui vont s'affirmer à partir de la fin du $\mathrm{xx}^{\mathrm{e}}$ et du début du XXI ${ }^{\mathrm{e}}$ siècle.

En fin de compte, s'agissant du standard minimum protecteur des intérêts des investisseurs étrangers, il sera repris dans tous les accords modernes d'investissements. La critique tiers-mondiste n'aura pas empêché sa reformulation. Il deviendra la norme du traitement juste et équitable. Plus qu'une norme, il s'apparentera de plus en plus à un dogme obligeant la surprotection inconditionnelle des investisseurs étrangers ${ }^{92}$.

En analysant en profondeur le standard minimum de traitement des étrangers en droit international, on a compris que la notion serait née sur le terrain controversé de la colonialité. Ce n'est donc pas un hasard si ce standard justifiait pendant longtemps différentes formes d'intervention, dont précisément la diplomatie de la canonnière. Cette pratique tant critiquée par le juriste latino-américain Carlos Calvo par ce qu'il en ressort pour ce dernier une volonté coloniale qui rappelle de vieilles expériences subies, a été pourtant "normale» au regard d'une bonne partie de la doctrine internationale et de la jurisprudence. Un élément implicite de colonialité s'incruste dans les relations entre les États, et marquera les destins du droit international. Cet élément de colonialité résidant dans le fait d'établir une approche du standard international qui ne tient compte que des pratiques des États civilisés (en l'occurrence les États occidentaux) servira pendant longtemps d'argumentaire à la fabrication d'une dynamique de la différence. L'Occident est effectivement au cœur de cette dynamique 
de la différence. Certes, au cours de son histoire le Japon entreprît une politique internationale fondée sur la logique du standard de civilisation qui reconnaissait comme sujets les États qui étaient assez civilisés pour assumer leur souverainetée ${ }^{93}$, on peut toutefois affirmer (sans nul doute) que cela n'a pas eu tellement de conséquences sur l'ordre économique global. Par exemple, aujourd'hui l'idée du traitement juste et équitable est l'héritière du standard occidental de civilisation dont les soubassements sont empreints de questions controversées. À l'heure où la protection des investisseurs étrangers se dessine comme une évidence dogmatique ${ }^{94}$, il ne semblerait pas fortuit de faire quelques fois un retour critique sur la genèse des standards internationaux de protection.

\section{NOTES}

1. Sur les rapports entre l'universalisation du droit international et le colonialisme, voir Antony Anghie, «Finding the Peripheries: Sovereignty and Colonialism in Nineteenth-Century International Law ", Havard International Law Journal, $\mathrm{n}^{\circ} 1$, hiver 1999, p. 1-71. Pour plus de renseignements sur l'histoire du droit international des investissements, voir Andrew Newcombe et Lluís Paradell, Law and Practice of Investments Treaties, Standards of Treatment, Alphen-sur-leRhin, Kluwer Law international, 2009. Voir également Charles Leben (dir.), Droit international des investissements et de l'arbitrage transnational, Paris, Pedone, 2015. Pour comprendre les premières justifications du standard minimum de traitement en droit international, voir notamment Elihu Root, "The Basis of Protection to Citizens residing Abroad ", The American Journal of International Law, n 3, juillet 1910, p. 517-528.

2. Voir Antony Anghie, art. cité, p. 1.

3. Eric Hobsbawm, The Age of Empire, 1875-1914, New York, Vintage Books, 1987.

4. Cette idée est généralement soutenue par quelques auteurs de la pensée décoloniale. Antony Anghie partage également ce point de vue (art. cité, p. 1-2).

5. Voir James Thuo Ghattii, "Imperialism, Colonialism and International Law», Buffalo Law Review, $\mathrm{n}^{\circ} 4$, janvier 2007.

6. CIJ, Affaire Ahmadou Sadio Diallo (République de Guinée c. République démocratique du Congo). Exceptions préliminaires, arrêt du 24 mai 2007, § 39.

7. Ibid.

8. Arnaud de Nanteuil, Droit international de l'investissement, Paris, Pedone, 2014, p. 23.

9. Selon l'expression du philosophe décolonial, Santiago Castro-Gomez (La hybris del punto cero: biopoliticas imperiales y coloniadad del poder en la Nueva Granada (1750-1810), Bogotá, Pontificia Universidad Javeriana, 2005).

10. Voir Ramon Grosfoguel, «Les implications des altérités épistémiques dans la redéfinition du capitalisme global. Transmodernité, pensée frontalière et colonialité globale », Multitudes, $\mathrm{n}^{\circ} 26$, 2006, p. 53.

11. Plusieurs auteurs admettent avec Aníbal Quijano que la modernité est indissolublement liée à la colonialité. Selon Walter Mignolo et Ramon Grosfoguel, par exemple, il n'y a pas de modernité sans colonialité et il n'y a pas de colonialité sans modernité.

12. Arturo Escobar et Eduardo Restrepo, "Anthropologies hégémoniques et colonialité », Cahiers des Amériques latines, $n^{\circ} 62,2009$, p. 83-95. Ces deux auteurs ont repris la définition donnée par 
Quijano. En accord avec cette définition, Ramon Grosfoguel souligne que « la colonialité se réfère à la continuité des formes de domination et d'exploitation après la disparition des administrations coloniales produites par les structures et les cultures hégémoniques du systèmemonde capitaliste / patriarcal moderne / colonial » (art. cité, p. 61).

13. . Gerrit Walter Gong qui a entrepris des études approfondies sur la norme de civilisation la décrivait comme un ensemble $d$ '«hypothèses tacites et explicites" constituant le critère permettant à une civilisation donnée de distinguer «ceux qui appartiennent à [sa] société particulière de ceux qui ne le sont pas" (notre traduction). De cette distinction découle évidemment une situation dans laquelle «les [nations] qui remplissent les critères ou exigences du standard de civilisation d'une société donnée sont regroupées dans son cercle de membres civilisés, tandis que les nations qui ne sont pas à la hauteur sont exclues en tant que non civilisées ou peut-être barbares " (notre traduction). Voir Gerrit Walter Gong, The Standard of "Civilization" in International Society, Oxford, Clarendon Press, 1984, p. 3.

14. Edwin Borchard énumère un ensemble de brillants juristes qui se mettent tous d'accord sur cet élément civilisationnel pour définir le standard minimum, voir « Minimum Standard of the Treatment of Aliens ", Michigan Law Review, $n^{\circ}$ 4, 1940, p. 446-461.

15. D'éminents juristes et hommes politiques ont admis, sans hésitation, que pour parler de standard minimum de traitement des étrangers en droit international il faut se référer à la pratique des États civilisés. Parmi eux, John Westlake précisait que le standard minimum constitue la norme sur laquelle doit être fondée toute procédure judiciaire et administrative. Il soutient que tous les peuples de la civilisation européenne sont d'accord là-dessus. Voir John Westlake, International Law, Cambridge, Cambridge University Press, 1904, p. 313.

16. Brett Bowden, «The Colonial Origins of International Law. European Expansion and the Classical Standard of Civilization ", Journal of the History of International Law, $\mathrm{n}^{\circ} 1,2005$, p. 2-3 (notre traduction).

17. Nous pensons précisément aux différents conflits qui ont eu lieu en Amérique latine et les interventions que cela a engendrées. Voir Charles Leben (dir.), op. cit.

18. L'expression est d'Antony Anghie. Voir son ouvrage majeur, Imperialism, Sovereignity and the Making of International Law, Cambridge, Cambridge University Press, 2004.

19. Antony Anghie, art. cité, p. 5.

20. Dans le domaine du droit international, les premières réactions au standard minimum de traitement trouvent leurs racines dans les œuvres d'Andres Bello (célèbre homme politique et intellectuel d'Amérique latine, 1781-1865) et de Carlos Calvo (brillant intellectuel, diplomate et théoricien de droit international, 1824-1906). Voir notamment Andres Bello, Derecho internacional, Obras completas, Santiago, Pedro G. Ramirez, 1886, vol.10; Carlos Calvo, Le droit international, Théorie et pratique, $5^{\mathrm{e}}$ éd., Paris, Rousseau, 1896, 6 vol. Plus récent, le livre de Mohammed Bedjaoui remet en cause l'idée d'un droit international déterminé fondamentalement par l'Occident (Pour un nouvel ordre économique international, Paris, UNESCO, 1978).

21. Il faut constamment rappeler que cette norme a servi d'étalon de mesure pour le traitement des investisseurs étrangers dans les territoires non occidentaux.

22. Charles Leben (dir.), op. cit., p. 1.

23. Ibid., p. 2-3.

24. Il est certain que pour comprendre cet aspect de la discussion, il ne faut pas se limiter au $\mathrm{XVII}^{\mathrm{e}}$ siècle, mais il faudrait remonter au Moyen Âge.

25. Les chercheurs appartenant au courant Third World Approchaes to International Law proposent tous une réponse à l'éthique coloniale et postcoloniale du droit international. Il s'agit d'un champ de réflexion dans lequel les arguments généralement soutenus visent à défaire la nature coloniale du droit international. Pour une discussion sur la question, voir Luis Eslava et Sundhya Pahuja, «Beyond the (Post)Colonial: TWAIL and the Everyday Life of International Law », Journal 
of Law and Politics in Africa, Asia and Latin America / Verfassung und Recht in Übersee (VRÜ), $\mathrm{n}^{\circ} 2,2012$, p. 195-221, https://ssrn.com/abstract=2034228.

26. Brett Bowden, art. cité, p. 4.

27. Cité par Edward B. Barbier, Scarcity and Frontiers, How Economies Have Developed through Natural Resource Exploitation, Cambridge, Cambridge University Press, 2011, p. 195 (notre traduction).

28. Brett Bowden, art. cité, p. 4.

29. L'historiographie du droit international retient les influences du Pape sur les juristes tels que Franciscus de Vitoria et Hugo Grotius, pères fondateurs du droit international.

30. Brett Bowden, art. cité, p. 4. Et citations suivantes du même paragraphe.

31. Ibid., p. 9.

32. Ibid. Voir aussi James Brown Scott, The Spanish Origin of International Law: Francisco de Vitoria and his law of nations, Oxford, Clarendon Press, 1932, p. IX.

33. Antony Anghie, op. cit., p. 15.

34. Brett Bowden, art. cité, p. 12. Et citation suivante.

35. Antony Anghie, op. cit., p. 22.

36. Ibid. Voir aussi Antony Anghie, "Francisco de Vitoria and the Colonial Origins of International Law », Social \& Legal Studies, $n^{\circ} 3,1996$, p. 327.

37. Pour une très impressionnante lecture critique sur les Lumières, voir Louis Sala-Molins, Le Code noir ou le calvaire du Canaan, Paris, PUF, 2018.

38. Ibid., p. XIII.

39. Ibid., p. XIV.

40. Pour une discussion sur la manière dont l'Europe continue de façonner le monde par ses concepts et catégories, voir Dipest Chakrabarty, Provincializing Europe: Postcolonial Thought and Historical Difference (new edition), Princeton, Princeton University Press, 2000.

41. Comme la plupart des juristes de l'époque, Thomas E. Lawrence a reproduit ce préjugé envers les États non occidentaux. Voir Les principes $d u$ droit international, Oxford, Imprimerie de l'Université, 1920, p. 17.

42. Antony Anghie, "Francisco de Vitoria and the Colonial Origins of International Law ", art. cité, p. 103.

43. Brett Bowden, art. cité, p. 17.

44. Ibid., p. 18. Et citations suivantes du paragraphe.

45. Voir Antony Anghie, Imperialism, Sovereignty and the Making of International Law, op. cit.

46. Antony Anghie, «Finding the peripheries: Sovereignty and Colonialism in the NineteenthCentury International Law », art. cité, p. 18-19.

47. Ibid.

48. Ibid., p. 20-22.

49. Ibid., p. 21. Et citation suivante.

50. Ibid. Voir aussi Henry Wheaton, Elements of International Law, George Crafton Wilson ed., The Carnegie Institution of Washington, 1964, p. 15-18.

51. Il s'agit d'une querelle opposant la Grande-Bretagne et la Grèce, dans laquelle les agissements britanniques suscitent des controverses en France et en Russie. David Pacifico (connu sous le nom de Don Pacifico) est un juif portugais. Né à Gibraltar en 1784, c'est un sujet britannique. Après avoir été consul du Portugal au Maroc (1835-1837), puis consul général en Grèce, il s'installe à Athènes en tant que marchand. En 1847, sa maison est incendiée lors d'une émeute antisémite, mais la police reste silencieuse. Pacifico demande une indemnisation au gouvernement grec avec l'appui du secrétaire britannique aux Affaires étrangères, Lord Palmerston. Palmerston envoie un escadron de marine pour bloquer la côte grecque (janvier 1850) et contraindre les Grecs à satisfaire les exigences de Pacifico. Cela provoque des protestations des Français et des Russes, avec lesquels la Grande-Bretagne partage un protectorat 
de la Grèce. Néanmoins, les Grecs finissaient par accepter le paiement de $4000 £$, bien que, en raison de la perte de certains papiers, la commission n'accorde à Pacifico que $150 €$.

52. L'ensemble du discours est disponible en ligne: http://en.wikisource.org/wiki/ Don_Pacifico_Speech (notre traduction).

53. Il faut souligner que l'idée de ce discours sera plus tard retrouvée dans l'arrêt Mavrommatis, CPJI, 30 août 1924, Affaire des concessions Mavrommatis en Palestine, Recueil des Arrêts, p. 12. Il a été affirmé que : "C'est un principe élémentaire du droit international que celui qui autorise l'État à protéger ses nationaux lésés par des actes contraires au droit international commis par un autre État, dont ils n'ont pu obtenir satisfaction par les voies ordinaires. En prenant fait et cause pour l'un des siens, en mettant en mouvement, en sa faveur, l'action diplomatique ou l'action judiciaire internationale, cet État fait, à vrai dire, valoir son droit propre, le droit qu'il a de faire respecter en la personne de ses ressortissants, le droit international. »

54. Voir Andrew Newcombe et Lluís Paradell, op. cit., p. 12.

55. Edwin Borchard, art. cité, p. 456. Et citations suivantes du paragraphe.

56. Commentant les définitions proposées par plusieurs juristes positivistes, Edwin Borchard a souligné que « la norme internationale est composée de principes généraux reconnus par le droit interne de pratiquement tous les pays civilisés, et [qu']il ne faut pas supposer que tout État normal le répudierait ou, si cela était possible, ne le respecterait pas » (ibid., p. 458). Il reconnaît que les «pratiques uniformes des pays civilisés sont des composantes essentielles du droit international [mondial]» (ibid., p. 448).

57. Dionisio Anzilotti, «La responsabilité internationale des États à raison des dommages soufferts par des étrangers ", RGDIP, t. XIII, 1906, p. 25.

58. Charles Leben (dir.), op. cit., p. 25.

59. Cité par Brett Bowden, art. cité, p. 19.

60. Edwin Borchard, art. cité, p. 448-449.

61. Andreas H. Roth, The Minimum Standard of International Law Applied to aliens, Leyde, A. W. Sijthoff, 1950, p. 91 (notre traduction).

62. Jeswald W. Salacuse, The Law of Investment Treaties, $2^{\mathrm{e}}$ éd., Oxford, Oxford University Press, p. 62.

63. John P. Grant et J. Craig Barker, Encyclopaedic Dictionary of International Law, $3^{\mathrm{e}}$ éd., Oxford, Oxford University Press, 2009 (notre traduction).

64. L'intrusion coloniale réside dans le fait d'obliger l'État non occidental accueillant l'étranger occidental à lui fournir le même traitement qu'il s'attendrait à recevoir en Occident (Antony Anghie, «Finding the Peripheries: Sovereignty and Colonialism in Nineteenth-Century International Law ", art. cité, p. 46).

65. Cité par Carlo Focarelli, International law as social construct, the struggle for global justice, Oxford, Oxford University Press, 2012, p. 167 (notre traduction).

66. Carlos Calvo, op. cit., t. I, p. 350.

67. Pour expliquer les interventions souvent infondées, Carlos Calvo a fait référence à la «différence du régime politique dans lequel sont placés les peuples de l'Amérique et les États européens ». Pour lui, les relations entre ces États de régions différentes et de statut différent (civilisé/non civilisé) évoquent des «traditions surannées du système colonial ", alors même qu'en droit international le principe de l'égalité entre les États a été établi (ibid., p. 349). Pour une actualité plus récente concernant l'intervention en droit international, voir CIJ, 9 avr.1949, Affaire du détroit de Corfu, fond, Recueil, 1949, p. 4. À la p. 35 de l'arrêt, on peut lire ce passage important : « Le prétendu droit d'intervention ne peut être envisagé par elle [la Cour] que comme la manifestation d'une politique de force, politique qui, dans le passé, a donné lieu aux abus les plus graves et qui ne saurait, quelles que soient les déficiences présentes de l'organisation internationale, trouver aucune place dans le droit international. »

68. Antony Anghie, Imperialism, Sovereignity and the Making of International Law, op. cit. 
69. Pour mieux comprendre les enjeux actuels liés à la colonialité, voir Walter Mignolo, Desobediencia epistémica. Retórica de la modernidad, lógica de la colonialidad y gramática de la descolonialidad, Buenos Aires, Ediciones del Signo, 2010.

70. Par exemple, les distinctions économique/public, public/privé, religieux/laïque, etc.

71. Voir Martti Koskenniemi, «Histories of International Law: Dealing with Eurocentrism ", Journal of the Max-Planck-Institute for European Legal History, n 19, 2011, p. 152-155. Pour revenir à la question de la civilisation, la dualité civilisé/non civilisé renvoie à "une hiérarchie épistémique où les connaissances occidentales sont privilégiées sur les cosmologies et sur les connaissances non occidentales ». Voir Ramon Grosfoguel, art. cité, p. 57.

72. C'est ainsi que le concept de traitement national formulé par les États latino-américains et caribéens comme norme de protection des étrangers a été écarté du droit international pour le simple fait qu'il ne reflétait pas l'idéal occidental. En ce sens, on peut interpréter le standard national comme une réaction à « l'universel manquant » qui caractérise le standard minimum de traitement.

73. Cette nouvelle rencontre s'affirme notamment par le biais de l'épistémè. C'est ce que Walter Mignolo appellerait une colonialité épistémique. Voir notamment son article, « Géo-politique de la sensibilité et du savoir. (Dé)colonialité, Pensée frontalière et Désobéissance épistémologique », Mouvements, $\mathrm{n}^{\circ} 73,2013$, p. 181-190.

74. Charles Leben (dir.), op. cit., p. 51.

75. CIJ, Affaire de la Barcelona Traction, opinion individuelle, Recueil, 1970, p. 246.

76. Charles Leben (dir.), op. cit., p. 9.

77. Voir Frank G. Dawson et Ivan L. Head, International Law, national tribunals and the right of aliens,

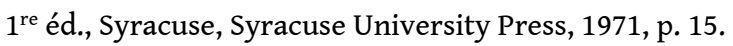

78. Muthucumaraswamy Sornarajah, The International Law on Foreign Investment, $2^{\mathrm{e}}$ éd., Cambridge, Cambridge University Press, 2004, p. 18-20. Voir aussi D. R. Shea, The Calvo Clause: A Problem of Inter-American and International Law and Diplomacy, Minnesota, University of Minnesota Press, 1955.

79. Voir Luis Drago, «Les emprunts d'État et leurs rapports avec la politique internationale », RGDIP, Paris, Pedone, 1907, p. 86.

80. L'ensemble de ces affirmations est surnommé « doctrine Calvo ».

81. Donald R. Shea, op. cit., p. 24-27.

82. CPJI, 30 août 1924, Affaire des concessions Mavrommatis en Palestine, op. cit.

83. Voir Frank Griffith Dawson, "The Influence of Andres Bello on the Latin-American Perceptions of Non-Intervention and State Responsibility ", British Yearbook of International Law, vol. 57, n 1, 1986, p. 285-287.

84. «El extranjero a su entrada contrae tacitamente la obligacion de sujetarse a las leyes i a la jurisdiccion local, i el estado le ofrece de la misma manera la proteccion de la autoridad publica, depositada en los tribunales " (Derecho internacional, Obras completas, op. cit., p. 118-119).

85. Voir Francisco V. Garcia-Amador, Louis B. Sohn and Richard R. Baxter, Recent Codification of the Law of State Responsibility for Injuries to Aliens, New York, Dobbs Fern-Leiden, 1974, p. 32.

86. Charles Leben (dir.), op. cit., p. 48.

87. Mohammed Bedjaoui, op. cit., p. 110.

88. Sentence Texaco Calasiatic c. Gouvernement libyen, 19 janvier 1977, § 85.

89. Charte des droits et devoirs économiques des États, 1974, art. 2, § 1.

90. Pour mieux comprendre le contexte des revendications tiers-mondistes, voir Mohammed Bendjaoui, op.cit. ; Ronald St. John Macdonald et Douglas Millar Jonhston, The Structure and Process of International Law: Essays in Legal Philosophy Doctrine and Theory, La Haye, Martinus Nijhoff, 1983 ; Ahmed Mahiou, «Les implications du nouvel ordre économique international », RBDI, 1976, p. 421-450 ; Burns H. Weston, «The New International Economic Order and the Deprivation of Foreign Proprietary Wealth: Reflections upon the Contemporary International Law Debate», 
dans Richard B. Lillich (dir.), International Law of State Responsibility for Injuries to Aliens, Charlottesville, University Press of Virginia, 1983, p. 89-148.

91. Charles Leben (dir.), "Les investissements miniers dans les pays en développement ", JDI, 1986, p. 898-902.

92. Pour une critique actuelle, voir Renaud Beauchard, L'assujettissement des Nations. Controverses autour du règlement des différends entre États et investisseurs, Paris, CharlesLéopold Mayer, 2017.

93. Sur cette question, voir par exemple Joshua A. Fogel, The Litterature of Travel in the Japan Rediscovery of China, 1862-1945, Stanford, Stanford University Press, 1996, p. 46-57 ; Kirk W. Larsen, Tradition, Treaties, and Trade. Qing Imperialism and Choson Korea, 1850-1910, Cambridge, Harvard University Press, 2008.

94. À défaut de ce traitement, un arbitrage investisseur-État peut être initié par l'investisseur.

\section{RÉSUMÉS}

Durant la fin du $\mathrm{XIX}^{\mathrm{e}}$ et le début du $\mathrm{xx}^{\mathrm{e}}$ siècles, le standard minimum de traitement des investisseurs étrangers peut se lire dans les œuvres des juristes positivistes comme une norme de civilisation, c'est-à-dire un dispositif civilisationnel qu'il faut coûte-que-coûte imposer dans les relations commerciales internationales. D'origine occidentale et judéo-chrétienne, l'impératif de civilisation qui fonde la définition du standard minimum de traitement postule une protection juridique eurocentrée rejetant les pratiques normatives non occidentales (considérées comme non civilisées). Par ainsi, s'est opérée une nouvelle forme d'intrusion coloniale qui a permis aux États occidentaux de raffermir leur hégémonie sur la scène internationale. Les perspectives tiersmondistes (l'hostilité latino-américaine d'origine Calvo, en particulier) ont proposé une autre logique de protection des étrangers pour contrecarrer la dynamique occidentale, mais elle ne deviendra pas hégémonique.

During the late nineteenth and early twentieth centuries, the minimum standard of treatment of foreign investors has been considered by positivist jurist as a standard of civilisation, which means a civilizational rule that was imposed in international trade relations. Coming from the Western, the civilization bases the definition of the minimum standard of treatment that postulates a eurocentric legal protection rejecting the non-occidental practices treated as uncivilized. As a result, a new form of colonial intrusion has taken place, allowing Western countries to strengthen their hegemony on the international stage. Third-world perspectives (Latin American hostility) have proposed another logic of protection of foreigners to thwart the Western dynamic, but have not become hegemonic.

\section{INDEX}

Mots-clés : traitement, étranger, international, civilisation, colonialisme

Keywords : treatment, foreigner, international, civilization, colonialism 
AUTEUR

MILCAR JEFF DORCE

Doctorant en droit public, CRDEI, Université de Bordeaux 